\title{
Facile synthesis of carbon nitride micro-/nanoclusters with photocatalytic activity for hydrogen evolution $\uparrow$
}

Cite this: $R S C A d v ., 2013,3,22988$

\author{
Jian Liu, ${ }^{* a}$ Jianhui Huang, ${ }^{b}$ Dariya Dontosova ${ }^{a}$ and Markus Antonietti ${ }^{a}$
}

Received 20th August 2013

Accepted 2nd October 2013

DOI: $10.1039 / c 3 r a 44490 b$

www.rsc.org/advances

Morphologically diverse carbon nitride micro-/nanoclusters (nanowires, nanoribbons, and nanosheets) were synthesized by polycondensation of cyanamide on diatom substrates. The formation mechanism is speculated to be one dimensional growth and two dimensional extensions on diatom frustules with high curvature. Platinum deposited micro-/nanoclusters showed good performance for photocatalytic hydrogen evolution.

Carbon nitride materials have attracted great interest from the scientific community due to their unique properties. Specially, graphitic carbon nitride is in the focus due to the newly disclosed photocatalytic properties. ${ }^{1}$ What is termed graphitic carbon nitride is usually an incompletely condensed $\mathrm{N}$-bridged "poly(tri-s-triazine)" polymer with heptazine building blocks, which is approximately abbreviated as $\mathrm{g}-\mathrm{C}_{3} \mathrm{~N}_{4}$. The facile synthesis and easy engineering properties render $\mathrm{g}-\mathrm{C}_{3} \mathrm{~N}_{4}$ very popular and promising in the field of visible-light-driven photocatalysis. However, simple solutions to improve the photocatalytic activity are always desired., ${ }^{2,3}$

Copolymerization of cyanamide (dicyandiamide or melamine) with other specific molecules usually leads to a redshift of the absorbance onset so that extended range of wavelengths can be used for light energy conversion., ${ }^{4,5}$ The preformed carbon nitride could also be coupled with other semiconductors or noble metals and/or graphene to form a heterojunction for improved photocatalytic efficiency. ${ }^{6-11}$ At the same time, orthogonal routes are compatible with all these efforts to synergistically maximize the overall improvement. ${ }^{\mathbf{1 2 - 1 4}}$ Nanostructuration serves the purposes of surface area enhancement, improvement of the light harvesting capability,

${ }^{a}$ Department of Colloid Chemistry, Max Planck Institute of Colloids and Interfaces, 14424 Potsdam, Germany. E-mail: Jian.Liu@mpikg.mpg.de

${ }^{b}$ Environmental and Life Sciences Department, Putian University, Putian 351100, P.R. China

$\dagger$ Electronic supplementary information (ESI) available: Experimental section, Fig. S1-S6 including SEM images, FTIR spectra and DRS spectra. See DOI: 10.1039/c3ra44490b as well as optimizing charge transport properties, which are all important factors for photocatalysis. ${ }^{15-20}$ Usually, templating method (soft and hard templating, including silica and bio-inspired templates) are employed to construct nanostructured carbon nitride materials..$^{21-27}$ The well-established nanowires, nanoribbons and nanosheets structures are excellent candidates for the photocatalytic applications due to the good light harvesting properties and facile charge separation functions, and constructing carbon nitride into these micro-/nanostructures might be promising for improved light energy conversion purposes. ${ }^{28-30}$ But until now, there are few reports about the direct formation of carbon nitride micro-/ nanoclusters for photocatalytic applications, and engineering continuously different carbon nitride nanomorphologies for photocatalytic applications is even rarer. ${ }^{31,32}$

In the present work, carbon nitride materials with various nanomorphologies are synthesized using diatom frustules as a substrate. ${ }^{33}$ The morphologies can be tuned by the composition ratios of precursors (cyanamide and diatom substrate) and the calcination temperature in an inert atmosphere. The micro-/ nanoclusters formation is shown to occur as one dimensional growth from topological defects intrinsic to a carbon nitride coating layer and further integration of parallel nanowires towards nanoribbons and nanosheets. The obtained carbon nitride micro-/nanostructures could be applied in water splitting experiments for hydrogen evolution, while it was not necessary to remove the diatom substrate for the photocatalytic application. ${ }^{25}$ With Pt nanoparticles in situ deposited onto the micro-/nanoclusters under white light excitation, the material demonstrates good photocatalytic activity in hydrogen evolution, using triethanolamine as sacrificial agent to scavenge the photogenerated holes.

The carbon nitride micro-/nanoclusters (abbreviated as CNMN) were synthesized by a solid "incipient wetness impregnation" method based on our previous work. ${ }^{25}$ The diatom frustules were evenly ground with cyanamide in a mortar by mechanical mixing. After polycondensation, the carbon nitride materials are obtained as yellow colored 
free-flowing powders which are not intergrown (see the large area image and optical digital image in Fig. S1 of ESI $\dagger$ ). The diatom frustule acts as the substrate for the CNMN growth. Analyzing from scanning electron microscope (SEM) images, a thin carbon nitride layer was firstly formed on the monomerwetted diatom frustule, which acts as nucleation sites for the defined nanostructure growth from the surface (see Fig. S2a of ESI $\dagger$ ). With different amounts of cyanamide in the starting mixture, these secondary structures turn into different morphologies at $600{ }^{\circ} \mathrm{C}$. For simplicity, the obtained samples were named as $\mathrm{CNMN}_{x}-T$, with $x$ representing the weight ratios between cyanamide and evenly ground with cyanamide in a mortar by mechanical mixing. After polycondensation, the carbon nitride materials are obtained as yellow colored freeflowing powders which are not intergrown (see the large area image and optical digital image in Fig. S1 of ESI $\dagger$ ). The diatom frustule acts as the substrate for the CNMN growth. Analyzing from scanning electron microscope (SEM) images, a thin carbon nitride layer was firstly formed on the monomer-wetted diatom frustule, which acts as nucleation sites for the defined nanostructure growth from the surface (see Fig. S2a of ESI $\dagger$ ). With different amounts of cyanamide in the starting mixture, these secondary structures turn into different morphologies at $600{ }^{\circ} \mathrm{C}$. For simplicity, the obtained samples were named as $\mathrm{CNMN}_{x}-T$, with $x$ representing the weight ratios between cyanamide and diatom, and $T$ denoting the polycondensation temperature, respectively. For a weight ratio of 0.25 (abbreviated as $\mathrm{CNMN}_{0.25}-600$ ), solely nanowires are found to grow from the diatom frustule in Fig. 1a. As the ratio increased to $0.5\left(\mathrm{CNMN}_{0.5}-600\right)$, nanowire clusters or thin ribbons became the majority event (as shown in Fig. 1b). As the ratio further increased to $1\left(\mathrm{CNMN}_{1}-600\right)$, thin nanosheets are found to have grown around the whole diatom structure (Fig. 1c). The bare diatom substrate is also presented in Fig. $1 \mathrm{~d}$ as a reference. It is interesting to see that micro-/nanoclusters mainly grow from highly bent areas of the substrate, which brings up the
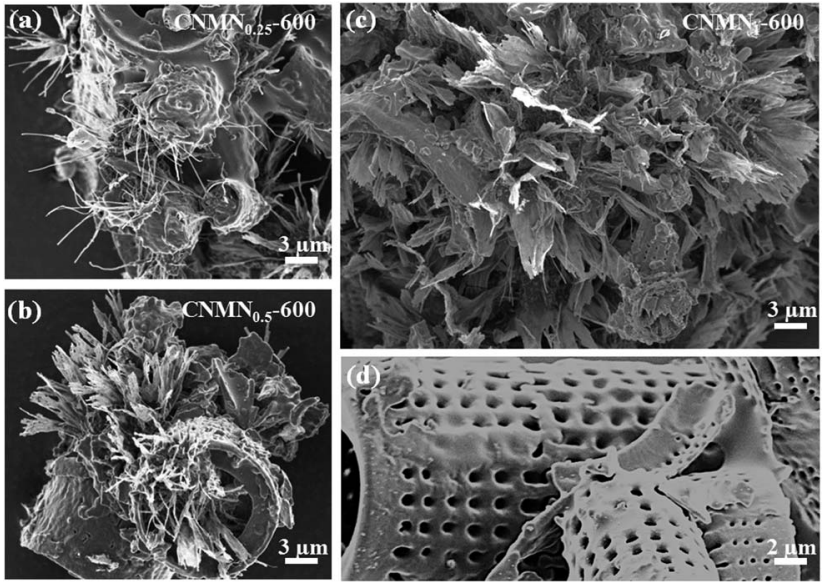

Fig. 1 SEM images of different carbon nitride micro-/nanoclusters condensed at $600^{\circ} \mathrm{C}$. (a) $\mathrm{CNMN}_{0.25}-600$ with nanowires as the major morphology; (b) $\mathrm{CNMN}_{0.5^{-}}$ 600 with nanoribbons as the majority; (c) CNMN-600 with nanosheets spreading along the whole diatom. Pure diatom in (d) was shown as reference, highlighting the big curvature of the frustule. interpretation that a defect induced secondary nucleation event drives the growth of these structures (presumably due to the high curvature of diatom frustule). ${ }^{27}$ The nanowires in $\mathrm{CNMN}_{0.25}-600$ have an average length of $8 \mu \mathrm{m}$ and are $250 \mathrm{~nm}$ in diameter. The nanowires are occasionally found to be curved, as illustrated in Fig. S2b of ESI. $\dagger$ It's worth mentioning that nanoribbons from $\mathrm{CNMN}_{0.5}-600$ and nanosheets from $\mathrm{CNMN}_{1}-600$ are of approximately same length, but different in size on the transverse direction. As the amount of cyanamide further increases (for example, $\mathrm{CNMN}_{2}-600$ or $\mathrm{CNMN}_{3}-600$ ), the obtained materials are intergrown with each other instead of constituting free-flowing powders and behave like bulk carbon nitride with embedded diatoms, as illustrated in Fig. S3 of ESI. $\dagger$ For $\mathrm{CNMN}_{2}-600$ or $\mathrm{CNMN}_{3}-600$, we can however still observe some porous structure from SEM images, which is presumably due to the gas evolution in the course of condensation. ${ }^{35}$ Therefore, the nanomorphologies of the micro-/nanoclusters could be controlled by the composition ratios between cyanamide and diatom substrate.

Fig. 2a show the Fourier transform infrared (FTIR) spectra of different $\mathrm{CNMN}_{x}-600(x=0.25,0.5$ and 1 , respectively), a composite spectrum of graphitic carbon nitride and the diatom substrate. The FTIR of $\mathrm{CNMN}_{1}-T$ is presented in Fig. S4a of ESI. $\dagger$ Typical aromatic C-N heterocyclic stretches in the 1100$1600 \mathrm{~cm}^{-1}$ spectral range and the breathing mode of the tri$s$-triazine units at $810 \mathrm{~cm}^{-1}$ demonstrate the formation of extended networks of $\mathrm{C}-\mathrm{N}-\mathrm{C}$ bonds. For X-Ray Diffraction (XRD) measurement, the diatom substrate was removed by $\mathrm{NH}_{4} \mathrm{HF}_{2}$ aqueous solution to release the carbon nitride phase. The SEM image of the released carbon nitride nanosheet $\left(\mathrm{CNMN}_{1}-600\right)$ is
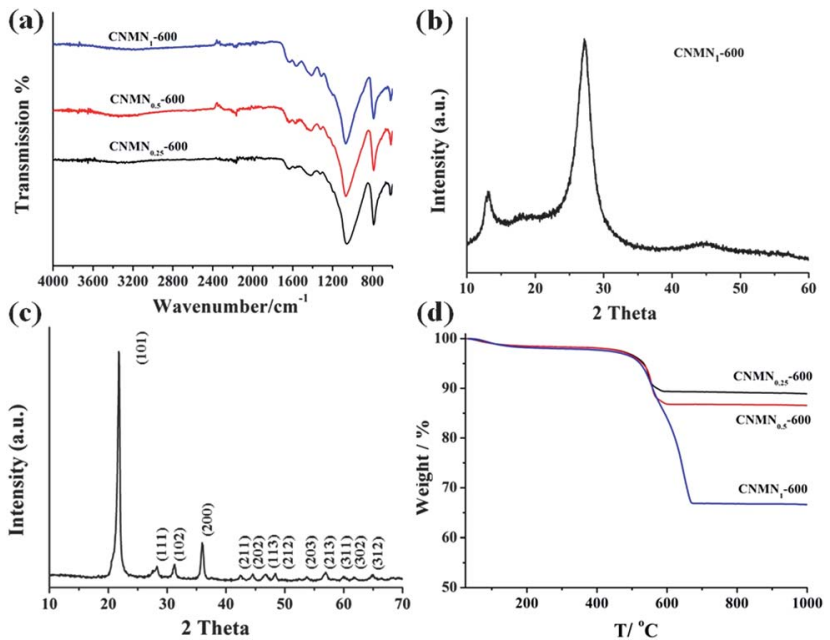

Fig. 2 Typical materials characterizations of obtained $C_{N M N}-T$. (a) FTIR of $\mathrm{CNMN}_{x}-600$ ( $x=0.25,0.5$ or 1 , respectively) showing typical C-N heterocycle stretches in the $1100-1600 \mathrm{~cm}^{-1}$ spectral range and the breathing mode of the tri-s-triazine units at $810 \mathrm{~cm}^{-1}$. (b) XRD of free standing CNMN after removal of diatom, with two peaks at $13.1^{\circ}$ and $27.3^{\circ}$ easily recognized due to the in-planar repeat period and stacking of the conjugated aromatic system, respectively. (c) XRD of the diatom substrate as reference, which is indexed to cristobalite according to the Miller indices labeled in the diffraction peaks. (d) TGA curves of $\mathrm{CNMN}_{x}-600$ show the carbon nitride contents are ca. $10 \%, 15 \%$ and $30 \%$, respectively. 
shown in Fig. S2c of the ESI. $\dagger$ Its XRD diffractogram (Fig. 2b) shows two characteristic peaks at $13.1^{\circ}$ and $27.3^{\circ}$ due to the inplanar repeat period and stacking of the conjugated aromatic system, respectively. In the composite sample, the diffractions of the diatom frustules are too strong to clearly identify the typical peaks of graphitic carbon nitride (Fig. S4b of ESI $\dagger$ ). The XRD of blank diatom is also included as references in Fig. 2c. The diffraction peaks of diatom labeled by their Miller indices are indexed to the cristobalite form of silica. ${ }^{36}$ Thermal gravimetric analysis (TGA) was used to determine the carbon nitride content in the CNMN/diatom composite (Fig. 2d). The corresponding carbon nitride contents in $\mathrm{CNMN}_{x}-600$ are $10 \%, 15 \%$, and $30 \%$, respectively, while the majority part of the composite is silica. $\mathrm{N}_{2}$ sorption measurement was performed to obtain BET surface areas of $\mathrm{CNMN}_{x}-600$ samples. The BET surface areas of $\mathrm{CNMN}_{0.25}-600, \mathrm{CNMN}_{0.5}-600$ and $\mathrm{CNMN}_{1}-600$ are 28, 27, and 24 $\mathrm{m}^{2} \mathrm{~g}^{-1}$, respectively. For comparison, the released carbon nitride sample $\left(\mathrm{CNMN}_{1}-600\right)$ after removal of diatom possesses a surface area of $57 \mathrm{~m}^{2} \mathrm{~g}^{-1}$.

In order to clarify temperature effects on the formation of the micro-/nanoclusters, different condensation temperatures were adopted. Mixtures of diatom and cyanamide with different ratios were calcined at $450{ }^{\circ} \mathrm{C}, 500{ }^{\circ} \mathrm{C}, 550{ }^{\circ} \mathrm{C}$ and $650{ }^{\circ} \mathrm{C}$, respectively. Table 1 summarizes the morphology developments of the micro- $/$ nanoclusters. Interestingly, micro-/nanostructures can be found already from $450{ }^{\circ} \mathrm{C}$ calcination temperature on, that is their nucleation starts already quite early in the temperature ramp. Higher than $650{ }^{\circ} \mathrm{C}$, the micro-/nanoclusters start to vanish and become smaller as compared to their appearance at lower temperatures, which illustrates the known thermal stability limit of carbon nitride. Different condensation temperatures in between render adjustment of the electronic and optical properties in combinations with the nanomorphologies possible, which is reflected in the diffuse reflectance spectra in Fig. S5a. $\dagger$ The absorption edge is redshifted with increasing condensation temperatures, demonstrating a lowered bandgap. ${ }^{1}$ At $650{ }^{\circ} \mathrm{C}$, the absorption edge of $\mathrm{CNMN}_{0.5}-650$ is blue-shifted again, which we attribute to the onset of depolymerization and decomposition of carbon nitride at these temperatures. The diffuse reflectance spectra of $\mathrm{CNMN}_{x}-650$ samples are also presented in Fig. S5b $\dagger$ and show that all three high temperature condensed samples derived from different cyanamide concentrations have the blueshifted bandgap.

Table 1 The morphology developments of $\mathrm{CNMN}_{x}-T$ related to the condensation temperatures, composition ratios between cyanamide and diatom ${ }^{a}$

\begin{tabular}{llllll}
\hline & $450{ }^{\circ} \mathrm{C}$ & $500{ }^{\circ} \mathrm{C}$ & $550{ }^{\circ} \mathrm{C}$ & $600{ }^{\circ} \mathrm{C}$ & $650{ }^{\circ} \mathrm{C}$ \\
\hline $\mathrm{CNMN}_{0.25}$ & $\checkmark$ & $\checkmark$ & $\checkmark$ & $\checkmark$ & $x$ \\
& Wire & Wire & Wire & Wire & \\
$\mathrm{CNMN}_{0.5}$ & $\checkmark$ & $\checkmark$ & $\checkmark$ & $\checkmark$ & $x$ \\
& Wire & Ribbon & Ribbon & Ribbon & \\
$\mathrm{CNMN}_{1}$ & $\checkmark$ & $\checkmark$ & $\checkmark$ & $\checkmark$ & $\checkmark$ \\
& Ribbon & Ribbon & Sheet & Sheet & Wire
\end{tabular}

${ }^{a}$ The " $\checkmark$ " indicates the obtained materials with corresponding morphology; while the " $X$ " indicates the material without micro- $/$ nanoclusters generated.
For $\mathrm{CNMN}_{0.25}-T$, the nanowire morphology prevails across different calcination conditions, except at highest temperature $\left(650{ }^{\circ} \mathrm{C}\right)$, as shown in Fig. S6 of ESI. $\dagger$ Interestingly, at the lowest examined temperature of $450{ }^{\circ} \mathrm{C}$, one dimensional growth of wire cores could be found (SEM image in Fig. S6a of ESI $\dagger$ ). This is presumably coupled to the polymerization of melem towards one dimensional polymer species, melon. ${ }^{34,37,38}$ No obvious differences of nanowires produced at different temperatures are recognized from the SEM images (Fig. S6a-d of ESI $\dagger$ ). Transmission electron microscopy (TEM) image of $\mathrm{CNMN}_{0.25}-600$ in Fig. S6f of $\mathrm{ESI} \dagger$ showed the nanowire grows outside from the diatom substrate.

With increasing amounts of cyanamide $\left(\mathrm{CNMN}_{0.5}-\mathrm{T}\right)$, the dominant morphology found in $\mathrm{CNMN}_{0.5}$ changes to nanoribbons, as illustrated in Fig. 3. Nanowires are still found in the low temperature polycondensation sample $\left(\mathrm{CNMN}_{0.5}-450\right.$ in Fig. 3a). Higher polycondensation temperatures (from $500{ }^{\circ} \mathrm{C}$ to $600{ }^{\circ} \mathrm{C}$ ) lead to nanoribbon morphologies, as illustrated in Fig. 3b-d. These nanoribbons are composed of several parallel nanowires, i.e. the extra monomer mass available is invested in parallel growth or secondary fiber nucleation, including some further integration processes. However, for $\mathrm{CNMN}_{0.5}-650$ (as illustrated in Fig. 3h), the carbon nitride coating was evenly distributed on the diatom surface without micro-/nanoclusters left, which agrees well with our previous results. ${ }^{25}$ Confocal microscopy was employed to investigate the photoluminescence of $\mathrm{CNMN}_{0.5}-600$, as illustrated in Fig. 3e and f. Under $488 \mathrm{~nm}$ laser excitation, the micro-/nanoclusters attached on the diatom frustule give luminescence. ${ }^{25}$ TEM image of $\mathrm{CNMN}_{0.5}-600$ in Fig. $3 \mathrm{~g}$ shows nanowire tips at the edge of nanoribbons, providing further experimental evidence that nanoribbons are combinations of nanowires.

Nanoribbons and nanosheets are finally common for $\mathrm{CNMN}_{1}-T$ samples, as seen from Fig. 4. Nanowires can also be occasionally observed as the building blocks for the nanoribbon and nanosheets. In the case of high cyanamide precursor concentration, low temperature condensations lead
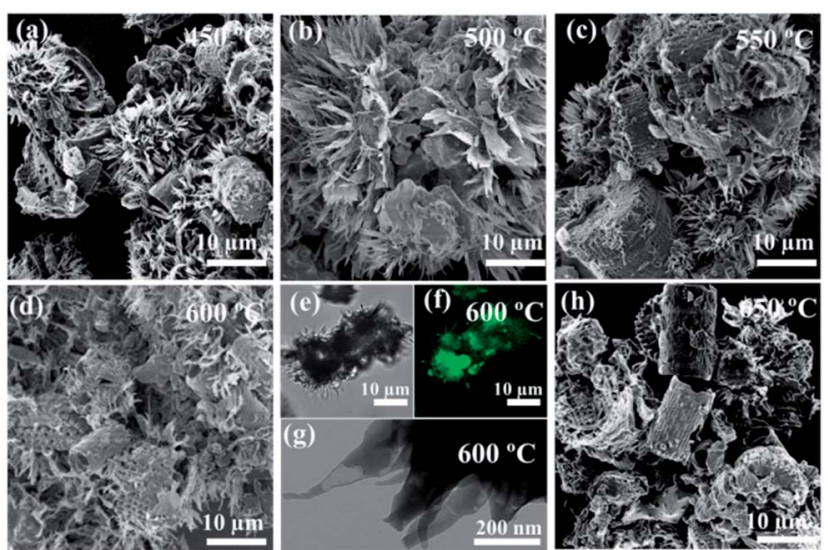

Fig. 3 SEM images of $\mathrm{CNMN}_{0.5}-\mathrm{T}$ synthesized at different condensation temperatures. (a) $450{ }^{\circ} \mathrm{C}$; (b) $500{ }^{\circ} \mathrm{C}$; (c) $550{ }^{\circ} \mathrm{C}$; (d) $600{ }^{\circ} \mathrm{C}$; (h) $650{ }^{\circ} \mathrm{C}$. (e and f) Optical and confocal fluorescence images of $\mathrm{CNMN}_{0.5}-600$ under laser excitation. (g) TEM of $\mathrm{CNMN}_{0.5}-600$ shows the nanoribbon edge was composed of nanowires. 

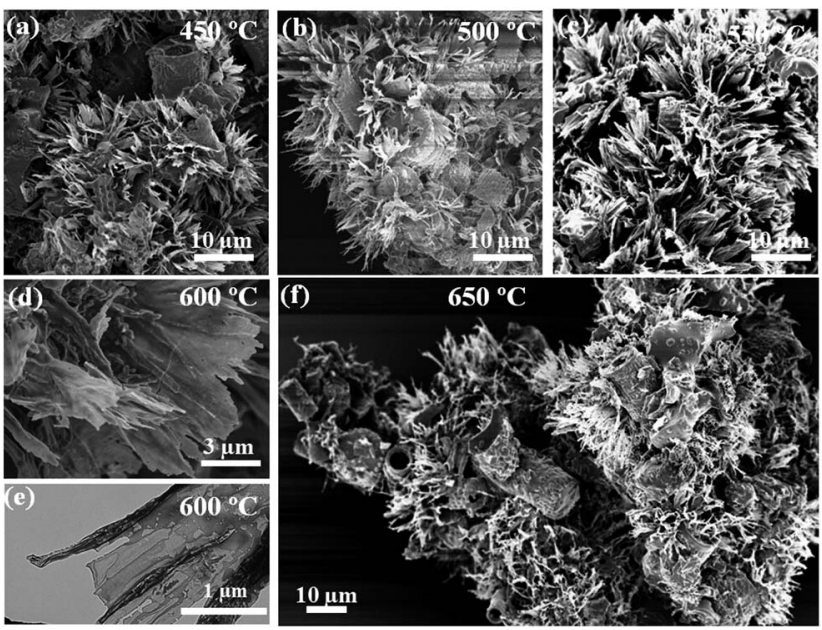

Fig. 4 SEM images of $\mathrm{CNMN}_{1}-T$ synthesized at different temperatures. (a) $450{ }^{\circ} \mathrm{C}$; (b) $500{ }^{\circ} \mathrm{C}$; (c) $550^{\circ} \mathrm{C}$; (d) $600{ }^{\circ} \mathrm{C}$; (f) $650{ }^{\circ} \mathrm{C}$. Nanoribbons and nanosheets are common morphologies for $\mathrm{CNMN}_{1}-\mathrm{T}$. (e) TEM image of $\mathrm{CNMN}_{1}-600$ showing the edge of nanosheet, in agreement with SEM analysis.

to nanoribbon morphologies (Fig. 4a and b), while at higher temperatures nanosheets prevail (Fig. $4 \mathrm{c}$ and d). TEM image of $\mathrm{CNMN}_{1}-600$ in Fig. $4 \mathrm{e}$ depicts the existence of two dimensional extended nanosheets with a width size of $3 \mu \mathrm{m}$, which matches well with the SEM observations. In the case of sample synthesized at $650{ }^{\circ} \mathrm{C}\left(\mathrm{CNMN}_{1}-650\right.$ in Fig. 4f), the width of micro-/ nanocluster is much smaller than that of its $600{ }^{\circ} \mathrm{C}$ calcination counterpart $\left(\mathrm{CNMN}_{1}-600\right)$, but the nanosheets are still there. We attribute this higher stability to the fact that the preceding nanosheets are better condensed and thereby more crystalline than fibers and ribbons.
All these data suggests the following formation mechanism. A thin carbon nitride layer was firstly formed on the diatom frustule, acting as nucleation sites for the subsequent micro-/ nanoclusters growth. The nanowires are firstly formed by one dimensional growth from topological defects of the layer. The formation mechanism is similar to the well-known vapor-solid growth of carbon nanotubes, however here without catalyst nanoparticles..$^{39,40}$ With higher precursor concentration and higher condensation temperature, the nanowires fuse together to form nanoribbons or nanosheets. Depolymerization of most structures at $650{ }^{\circ} \mathrm{C}$ is in agreement with the decomposition temperature of carbon nitride reported in the literature. ${ }^{31} \mathrm{Up}$ to $600{ }^{\circ} \mathrm{C}$, the band gaps of CNMNs decrease due to an increased condensation degree enabled by the increased temperatures. The whole process is schematically illustrated in Scheme 1.

For a demonstration of potential applications, the $\mathrm{CNMN}_{x^{-}}$ 600 materials were tested in a visible light driven water splitting assay for hydrogen evolution. Pt nanoparticles were in situ deposited onto the $\mathrm{CNMN}_{x}-600$ to perform photocatalytic hydrogen evolution from water in the presence of triethanolamine as a sacrificial agent. The amount of active carbon nitride was kept the same to allow direct comparison. The hydrogen evolution rates for $\mathrm{CNMN}_{0.25}{ }^{-600}, \mathrm{CNMN}_{0.5}-600$ and $\mathrm{CNMN}_{1^{-}}$ 600 are $4.1,6.1$ and $4.9 \mu \mathrm{mol} \mathrm{h}^{-1}$, respectively (see Fig. 5). The nanoribbon has the best performance among there micro-/ nanostructures, which is thought to be due to the combination of light harvesting property and good charge separation in two dimensional nanoribbon..$^{30}$ The amount of photogenerated hydrogen increased linearly with the illumination time during ten hours run experiment. For comparisons, bulk carbon nitride (bulk $\mathrm{CN}$ ) and mesoporous carbon nitride (mesoporous CN) were also employed for photocatalytic water splitting.

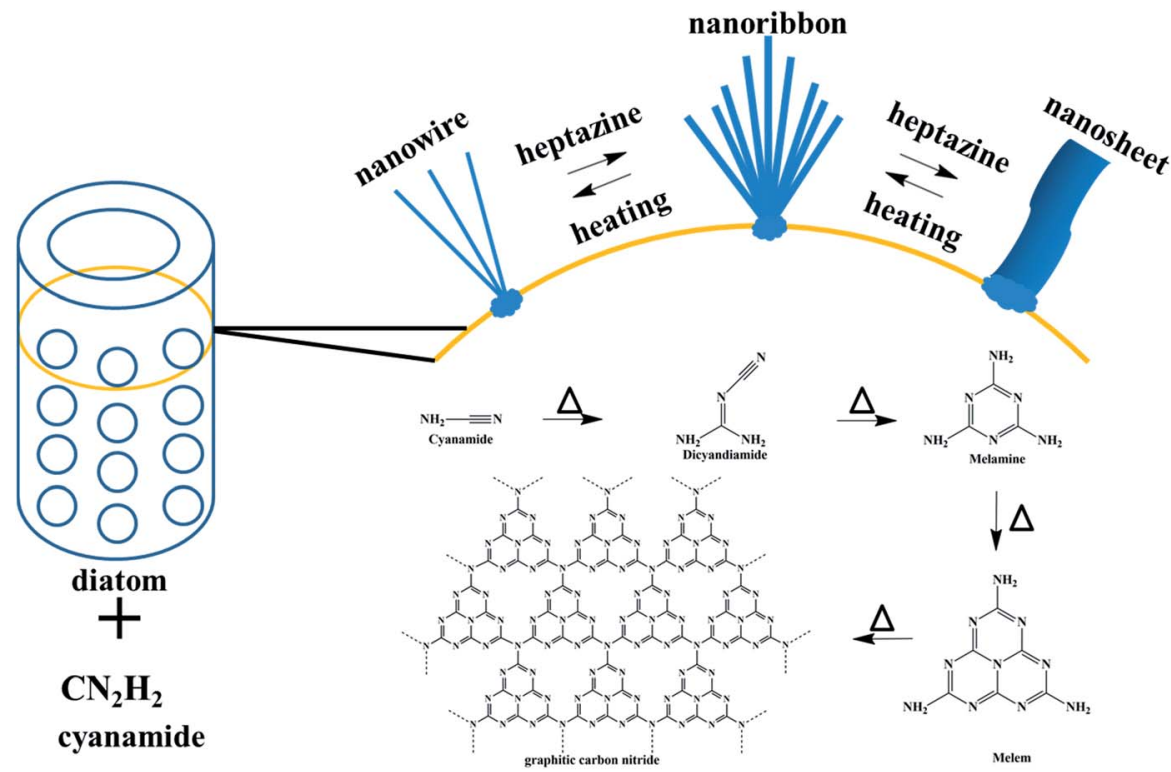

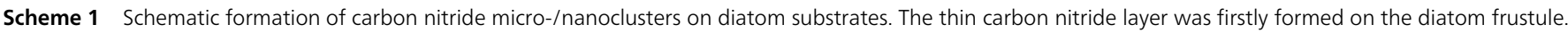

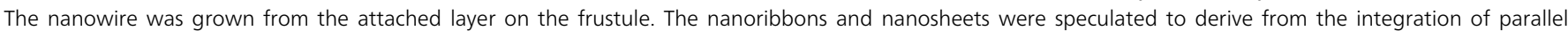

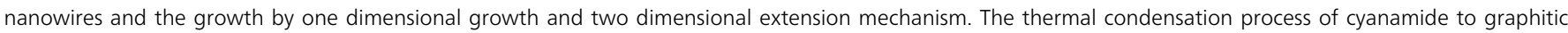
carbon nitride was also included in the scheme. 


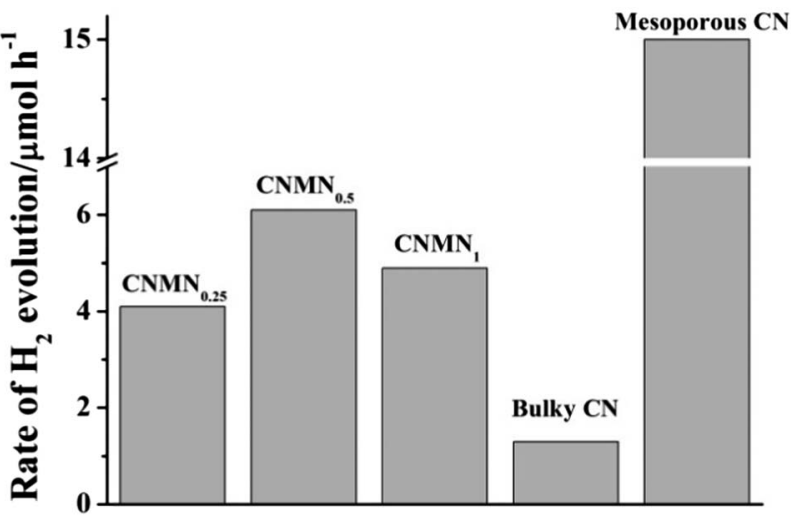

Fig. 5 Histogram of hydrogen evolution rate using different $\mathrm{CNMN}_{x}-600$ $\left(\mathrm{CNMN}_{0.25}-600, \mathrm{CNMN}_{0.5}-600\right.$ and $\left.\mathrm{CNMN}_{1}-600\right)$; values for bulky carbon nitride and mesoporous carbon nitride were shown as references. The active carbon nitride was kept at $50 \mathrm{mg}$ in the experiment.

Compared with the bulk sample, the CNMNs perform much better in hydrogen evolution efficiencies. The enhancement ratios of $\mathrm{CNMN}_{x}-600$ compared to bulky sample are 3.2, 4.6 and 3.8 times, respectively. The mesoporous material however gives the best performance due to its very large surface area. Further increasing the surface area of micro-/nanoclusters would obviously be meaningful to further improve the activity.

\section{Conclusions}

In conclusion, carbon nitride materials with different morphologies such as nanowires, nanoribbons and nanosheets were obtained by the in situ thermal condensation of cyanamide onto diatom substrates. Morphologies were controlled by the weight ratios between cyanamide and the diatom substrate and the condensation temperatures. The formation mechanism was ascribed to the one dimensional growth and two dimensional extensions of carbon nitride nanostructures, nucleated from positions on the diatom substrate with high curvature. The nanostructures obtained here demonstrate good photocatalytic activity for water splitting. Among the three nanomorphologies, nanoribbons give the best photocatalytic performance, which was attributed to the combination of good light harvesting property and efficient charge separation in two dimensional nanoribbons. The facile synthesis routes presented here is in principle compatible with other possibilities for engineering the carbon nitride with different properties for improved photocatalysis, and the relevant work is under progress.

\section{Acknowledgements}

J. Liu acknowledges the support of Alexander von Humboldt Foundation. Dr Hongqiang Wang and Dr Qianling Cui of MPIKG are appreciated for experimental assistance. J. Huang acknowledges the National Natural Science Foundation of China (21103095) and the Natural Science Foundation of Fujian Province (2010J05030).

\section{Notes and references}

1 X. Wang, K. Maeda, A. Thomas, K. Takanabe, G. Xin, J. M. Carlsson, K. Domen and M. Antonietti, Nat. Mater., 2008, 8, 76.

2 K. K. Han, C. C. Wang, Y. Y. Li, M. M. Wan, Y. Wang and J. H. Zhu, RSC Adv., 2013, 3, 9465.

3 L. Huang, Y. Li, H. Xu, Y. Xu, J. Xia, K. Wang, H. Li and X. Cheng, $R S C A d v$., 2013, DOI: 10.1039/c3ra42712a.

4 J. Zhang, X. Chen, K. Takanabe, K. Maeda, K. Domen, J. D. Epping, X. Fu, M. Antonietti and X. Wang, Angew. Chem., Int. Ed., 2010, 49, 441.

5 J. Zhang, G. Zhang, X. Chen, S. Lin, L. Möhlmann, G. Dołęga, G. Lipner, M. Antonietti, S. Blechert and X. Wang, Angew. Chem., 2012, 124, 3237.

6 X.-H. Li, X. Wang and M. Antonietti, Chem. Sci., 2012, 3, 2170. 7 X.-H. Li, J.-S. Chen, X. Wang, J. Sun and M. Antonietti, J. Am. Chem. Soc., 2011, 133, 8074.

8 A. Du, S. Sanvito, Z. Li, D. Wang, Y. Jiao, T. Liao, Q. Sun, Y. H. Ng, Z. Zhu and R. Amal, J. Am. Chem. Soc., 2012, 134, 4393.

9 S. Yan, S. Lv, Z. Li and Z. Zou, Dalton Trans., 2010, 39, 1488. 10 Y. Wang, X. Bai, C. Pan, J. He and Y. Zhu, J. Mater. Chem., 2012, 22, 11568.

11 J. Liu, M. Li, J. Zhou, C. Ye, J. Wang, L. Jiang and Y. Song, Appl. Phys. Lett., 2011, 98, 023110.

12 S. Yang, Y. Gong, J. Zhang, L. Zhan, L. Ma, Z. Fang, R. Vajtai, X. Wang and P. M. Ajayan, Adv. Mater., 2013, 25, 2452.

13 Q. Xiang, J. Yu and M. Jaroniec, J. Phys. Chem. C, 2011, 115, 7355.

14 S. Yang, W. Zhou, C. Ge, X. Liu, Y. Fang and Z. Li, RSC Adv., 2013, 3, 5631.

15 J. Liu, G. Liu, M. Li, W. Shen, Z. Liu, J. Wang, J. Zhao, L. Jiang and Y. Song, Energy Environ. Sci., 2010, 3, 1503.

16 H. Zhou, X. Li, T. Fan, F. E. Osterloh, J. Ding, E. M. Sabio, D. Zhang and Q. Guo, Adv. Mater., 2010, 22, 951.

17 J. Liu, Q. Yang, W. Yang, M. Li and Y. Song, J. Mater. Chem. A, 2013, 1, 7760.

18 S. Liu, L. Wang, J. Tian, J. Zhai, Y. Luo, W. Lu and X. Sun, RSC Adv., 2011, 1, 951.

19 J. Liu, M. Li, J. Wang, Y. Song, L. Jiang, T. Murakami and A. Fujishima, Environ. Sci. Technol., 2009, 43, 9425.

20 J. Liu, Q. Yang, M. Li, w. Zhu, H. Tian and Y. Song, Philos. Trans. R. Soc., A, 2013, 371, 20120314.

21 Y. Wang, X. Wang, M. Antonietti and Y. Zhang, ChemSusChem, 2010, 3, 435.

22 M. Groenewolt and M. Antonietti, Adv. Mater., 2005, 17, 1789.

23 X. Chen, Y.-S. Jun, K. Takanabe, K. Maeda, K. Domen, X. Fu, M. Antonietti and X. Wang, Chem. Mater., 2009, 21, 4093.

24 X.-H. Li, J. Zhang, X. Chen, A. Fischer, A. Thomas, M. Antonietti and X. Wang, Chem. Mater., 2011, 23, 4344.

25 J. Liu and M. Antonietti, Energy Environ. Sci., 2013, 6, 1486. 26 K. Kailasam, J. D. Epping, A. Thomas, S. Losse and H. Junge, Energy Environ. Sci., 2011, 4, 4668.

27 S. S. Park, S.-W. Chu, C. Xue, D. Zhao and C.-S. Ha, J. Mater. Chem., 2011, 21, 10801. 
28 P. Yang, MRS Bull., 2012, 37, 806.

29 Y. Ide, M. Matsuoka and M. Ogawa, J. Am. Chem. Soc., 2010, 132, 16762.

30 Q. Liu, Y. Zhou, J. Kou, X. Chen, Z. Tian, J. Gao, S. Yan and Z. Zou, J. Am. Chem. Soc., 2010, 132, 14385.

31 Y. Cui, Z. Ding, X. Fu and X. Wang, Angew. Chem., Int. Ed., 2012, 51, 11814.

32 X. Bai, L. Wang, R. Zong and Y. Zhu, J. Phys. Chem. C, 2013, 117, 9952.

33 C. Sanchez, H. Arribart and M. M. G. Guille, Nat. Mater., 2005, 4, 277.

34 Y. Zhao, Z. Liu, W. Chu, L. Song, Z. Zhang, D. Yu, Y. Tian, S. Xie and L. Sun, Adv. Mater., 2008, 20, 1777.
35 B. V. Lotsch, M. Döblinger, J. Sehnert, L. Seyfarth, J. Senker, O. Oeckler and W. Schnick, Chem.-Eur. J., 2007, 13, 4969.

36 Y. Jia, W. Han, G. Xiong and W. Yang, J. Colloid Interface Sci., 2008, 323, 326.

37 Z. Zhang, H. Yuan, J. Zhou, D. Liu, S. Luo, Y. Miao, Y. Gao, J. Wang, L. Liu and L. Song, J. Phys. Chem. B, 2006, 110, 8566.

38 A. Thomas, A. Fischer, F. Goettmann, M. Antonietti, J.-O. Müller, R. Schlögl and J. M. Carlsson, J. Mater. Chem., 2008, 18, 4893.

39 Z. W. Pan and Z. L. Wang, Science, 2001, 291, 1947.

40 J. L. Johnson, Y. Choi and A. Ural, J. Vac. Sci. Technol., B: Microelectron. Nanometer Struct.-Process., Meas., Phenom., 2008, 26, 1841. 